\title{
BSM Higgs results from ATLAS and CMS
}

\author{
Martin Flechl ${ }^{1, a}$ on behalf of the ATLAS and CMS collaborations \\ ${ }^{1}$ Albert-Ludwigs-Universität Freiburg, Physikalisches Institut, Hermann-Herder-Str. 3, 79104 Freiburg, Germany
}

\begin{abstract}
Searches for Higgs bosons in different extensions of the Standard Model (SM) are presented. These include the Minimal Supersymmetric extension of the SM (MSSM), the next-to-MSSM (NMSSM), models with additional scalar singlets, doublets, or triplets, and generic searches for models with couplings modified with respect to the SM or for non-SM Higgs boson decay channels. Results are based on data collected by the ATLAS and CMS experiments in 2011 and 2012 at the LHC. No excess is found in any of the searches and thus the resulting exclusion limits are given.
\end{abstract}

\section{Introduction}

A large number of searches for beyond-the-SM (BSM) Higgs bosons have been performed by the ATLAS [1] and CMS [2] experiments at the LHC [3], using collision data at a center-of-mass energy of $7 \mathrm{TeV}$ (2011) and $8 \mathrm{TeV}$ (2012). The discovery of a particle with a mass of $125 \mathrm{GeV}$ compatible with a Higgs boson by the ATLAS and CMS experiments [4, 5] leads to new questions - in particular whether it is the SM Higgs boson or one of typically many Higgs bosons predicted by extensions of the Standard Model. Experimentally, this implies measuring the properties of the new boson (not covered here) and searching for additional Higgs bosons. In the following an overview of the most recent results of searches for BSM Higgs bosons using an integrated luminosity of at least $5 \mathrm{fb}^{-1}$ is presented.

\section{MSSM Higgs boson searches}

The MSSM employs a type-II 2-Higgs doublet model (2HDM), which predicts the existence of three neutral Higgs bosons $(h, H, A)$ and a charged pair $\left(H^{ \pm}\right)$. At treelevel, the Higgs sector of the MSSM can be described by only two additional parameters, e.g. by one of the masses of the heavy Higgs bosons and $\tan \beta$, the ratio of the vacuum expectation values of the two Higgs doublets. In the MSSM, the recently discovered boson with $m \approx$ $125 \mathrm{GeV}$ can be identified with the $h$ boson for most of the $m_{A}-\tan \beta$ region not excluded by direct MSSM Higgs boson searches and also with the $H$ particle if the charged Higgs boson mass is below $150 \mathrm{GeV}$ and $\tan \beta$ is small [6]. Since the couplings of the MSSM Higgs bosons to $b$ and $t$ quarks and tau leptons are enhanced for large $\tan \beta$ most

\footnotetext{
a e-mail: martin.flechl@cern.ch
}

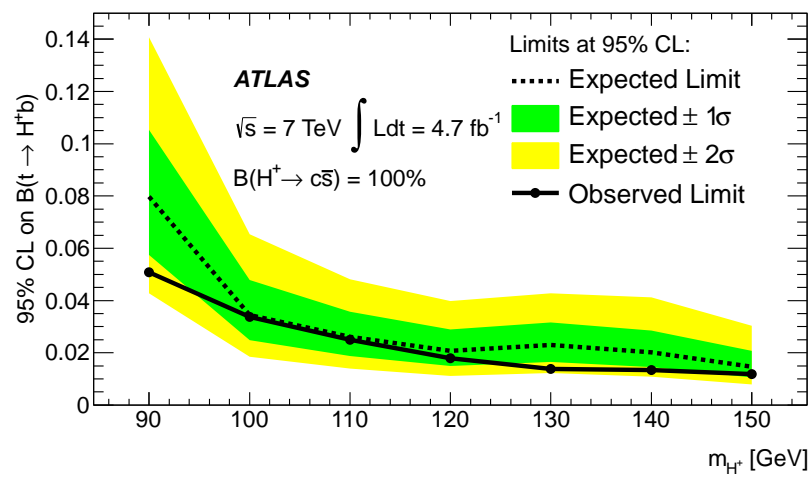

Fig. 1. ATLAS exclusion limit on $\mathrm{B}\left(t \rightarrow b H^{+}\right)$for charged Higgs boson decays to $c \bar{s}$ [7], assuming $\mathrm{B}\left(H^{+} \rightarrow c \bar{s}\right)=100 \%$.

searches for these bosons focus on Higgs boson production in association with $b$ and $t$ quarks and decay modes involving $b, t$ and $\tau$ particles.

\section{$2.1 H^{+} \rightarrow c \bar{s}$}

ATLAS searches for charged Higgs bosons produced in semileptonic $t \bar{t}$ events with $t \rightarrow b H^{ \pm}$and $H^{ \pm} \rightarrow c \bar{s}$ in $4.7 \mathrm{fb}^{-1}$ of $7 \mathrm{TeV}$ data [7]. The analysis requires an isolated lepton, 4 jets (two of them $b$-tagged), missing transverse energy $\left(E_{T}^{\text {miss }}\right)$ greater than 20 (30) $\mathrm{GeV}$ and the transverse mass $m_{T}$ of the lepton and $E_{T}^{\text {miss }}$ to satisfy $m_{T}>30 \mathrm{GeV}\left(m_{T}+E_{T}^{\text {miss }}>60 \mathrm{GeV}\right)$ in the muon (electron) channel. A kinematic fit is applied to the remaining events with the goal to find a second mass peak in the dijet distribution due to $H^{+} \rightarrow c \bar{s}$ events. Good agreement between data and SM expectation is observed, leading to a limit on the branching ratio $\mathrm{B}\left(t \rightarrow b H^{+}\right)=(5-1) \%$ for $m_{H^{ \pm}}=90-150 \mathrm{GeV}$, assuming $\mathrm{B}\left(H^{+} \rightarrow c \bar{s}\right)=100 \%$, as shown in figure 1. 


\section{$2.2 H^{+} \rightarrow \tau v$}

Both ATLAS and CMS search for charged Higgs bosons produced in $t \bar{t}$ events that decay to $H^{+} \rightarrow \tau v$. The ATLAS analysis [8] is based on $4.6 \mathrm{fb}^{-1}$ of $7 \mathrm{TeV}$ data and studies the final states $\tau_{\text {lep }}+$ jets, $\tau_{\text {had }}+$ lepton and $\tau_{\text {had }}+$ jets where the subscript indicates whether the tau lepton decays to leptons or hadrons+neutrino. The $\tau_{\text {had }}+$ jets channel is the most sensitive one for most of the $\mathrm{H}^{+}$mass range. The selection starts with a $\tau+E_{T}^{\text {miss }}$ trigger and requires the presence of exactly one hadronically decaying tau lepton, at least four jets (at least one of them $b$-tagged), no electrons or muons, $E_{T}^{\text {miss }}>65 \mathrm{GeV}$, a high significance of the missing transverse energy (quantified by its ratio to the square root of the sum of transverse momenta of reconstructed tracks) and a topology consistent with the presence of a hadronic top quark decay. The final discriminant used is a transverse mass built from the visible tau lepton decay products and the missing momentum vector. All backgrounds are estimated in a data-driven way by replacing muons in $\mu+$ jets collision data with simulated tau leptons for backgrounds with actual $\tau$ leptons, and by deriving data-driven corrections for the misidentification probabilities for backgrounds where jets or electrons are misidentified as $\tau$ leptons.

For all final states, the data agree with the SM expectation and an upper limit of $\mathrm{B}\left(t \rightarrow b H^{+}\right)=(5-1) \%$ assuming $\mathrm{B}\left(H^{+} \rightarrow \tau v\right)=100 \%$ is set for $m_{H^{ \pm}}=90-160 \mathrm{GeV}$. In a different approach, lepton universality in $t \bar{t}$ events is tested by observing the ratio of event yields with electrons or muons and with and without tau leptons in the final state which would be enhanced by the presence of a charged Higgs boson [9]. This brings down the limit after combining with the $\tau_{\text {had }}+$ jets final state to about $\mathrm{B}\left(t \rightarrow b H^{+}\right)=(3-1) \%$. Interpreting this limit in the context of the $m_{h}^{\max }$ of the MSSM [10], a large portion of the parameter space for a light $H^{ \pm}$can be excluded, see figure 2.

The CMS search [11] uses $2.2-4.9 \mathrm{fb}^{-1}$ of $7 \mathrm{TeV}$ data in the final states: $\tau_{\text {had }}+$ lepton, $e+\mu$, and $\tau_{\text {had }}+$ jets. Good agreement between data and expectation is observed and upper limits of $\mathrm{B}\left(t \rightarrow b H^{+}\right)=(3-2) \%$ for $m_{H^{ \pm}}=$ $80-160 \mathrm{GeV}$ are set.

\section{$2.3 \Phi \rightarrow b b$}

In the MSSM, the mode $\Phi \rightarrow b b(\Phi=h, H, A)$ is dominant for most regions of the parameter space. The CMS search [12] uses up to $4.8 \mathrm{fb}^{-1}$ of $7 \mathrm{TeV}$ data and focuses on $b$-associated production, $p p \rightarrow b \Phi \rightarrow b b b$, in two separate channels: The first requires three tight $b$-tagged jets and is split into two analyses searching for $m_{\Phi}$ either below or above $180 \mathrm{GeV}$. The second employs looser $b$-tagging requirements but demands that a muon overlaps with the leading $b$-tagged jet. The final discriminant is the invariant mass of the two leading $b$-tagged jets. Backgrounds are estimated from data, using control samples with 1 or 2 $b$-tagged jets. No excess is observed and combined limits exclude values of $\tan \beta>20-30$ for $m_{A}=90-350 \mathrm{GeV}$ in the MSSM ( $m_{h}^{\max }$ scenario), as shown in figure 3.

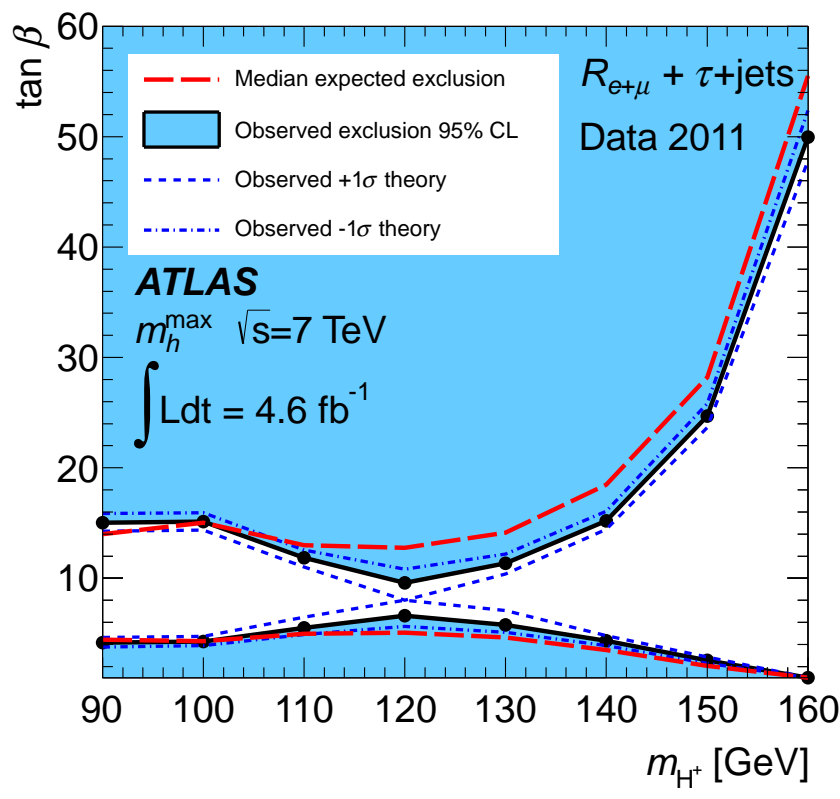

Fig. 2. ATLAS exclusion limit in the $m_{H^{ \pm}}-\tan \beta$ plane for the $m_{h}^{\max }$ scenario of the MSSM from the search for charged Higgs boson production in $t \bar{t}$ events and decays to $\tau \nu$. The limits are derived using $\tau_{\text {had }}+$ jets events and studying lepton universality [9].

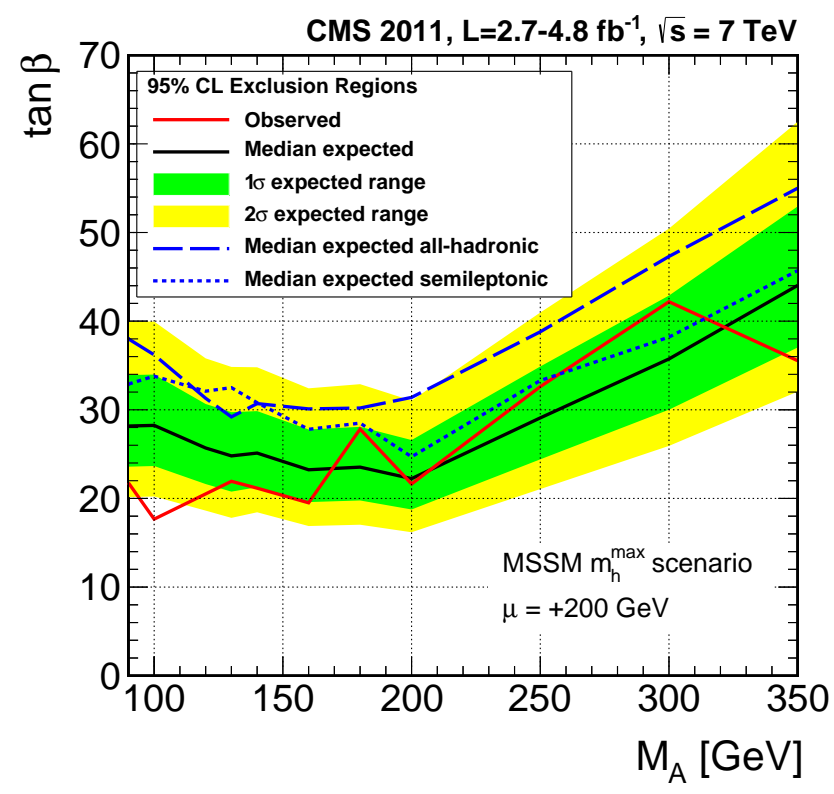

Fig. 3. CMS exclusion limit for Higgs boson decays via $H \rightarrow b b$ in $b$-associated production in the $m_{h}^{\max }$ scenario of the MSSM [12].

\section{$2.4 \Phi \rightarrow \tau \tau, \Phi \rightarrow \mu \mu$}

The CMS analysis uses $17 \mathrm{fb}^{-1}$ of $7 \mathrm{TeV}$ and $8 \mathrm{TeV}$ data to search for MSSM Higgs boson decays to a tau lepton pair [13], and $5 \mathrm{fb}^{-1}$ of $7 \mathrm{TeV}$ data for decays to a muon pair [14]. For the former, three different combinations of tau lepton decays are considered: $\tau_{\text {lep }} \tau_{\text {had }}, \tau_{\mathrm{e}} \tau_{\mu}$ and $\tau_{\mu} \tau_{\mu}$. The ATLAS analysis [15] in addition studies 


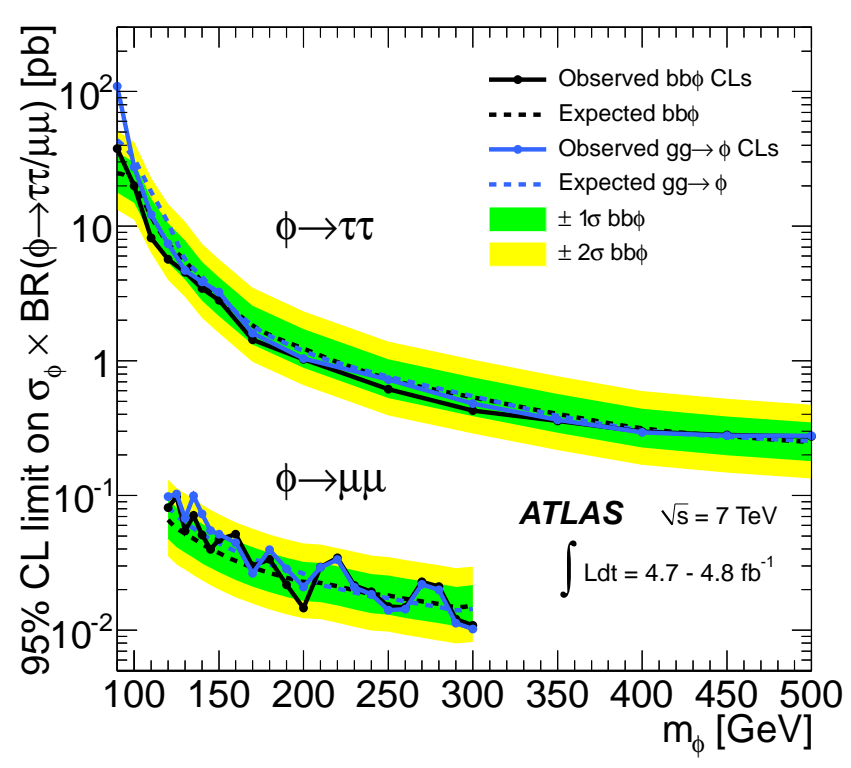

Fig. 4. ATLAS cross section times branching ratio limits for $g g$-fusion and $b$-associated production of Higgs bosons and subsequent decay into tau lepton or muon pairs in the $m_{h}^{\max }$ scenario of the MSSM [15].

the $\tau_{\text {had }} \tau_{\text {had }}$ final state, but does not use $\tau_{\mu} \tau_{\mu}$. For both ATLAS and CMS, each final state is split into two categories with and without additional $b$-tagged jets aiming to exploit the different production mechanisms ( $b$-associated and $g g$-fusion). The $b$-tagged categories provide a higher signal-over-background ratio but a smaller number of expected signal events. The final discriminant in all cases is an estimator, "Missing Mass Calculator" for ATLAS and "SVFit" for CMS, for the ditau mass based on different algorithms for finding the most likely value given the unknowns due to the neutrinos in the decay chain. The main background in all categories are $Z \rightarrow \tau \tau$ events which are estimated using a technique replacing muons in $Z \rightarrow \mu \mu$ enhanced collision data by simulated tau leptons. Most other relevant backgrounds are normalized in control regions.

No significant excess is observed and exclusion limits are produced. ATLAS provides model-independent cross section limits for both gluon-gluon fusion and $b$-quark associated production (see figure 4); and both experiments interpret their results in the context of the $m_{h}^{\max }$ scenario of the MSSM (the CMS limit is shown in figure 5). CMS excludes $m_{A}<125 \mathrm{GeV}$ (in the region not yet excluded by LEP) as well as values of $\tan \beta>5$ for $m_{A}<225 \mathrm{GeV}$; the exclusion region extends up to $m_{A}=800 \mathrm{GeV}$ and $\tan \beta>50$.

\section{$2.5 H \rightarrow W W$}

ATLAS searches for additional CP-even neutral Higgs bosons in a $2 \mathrm{HDM}$, assuming that the observed boson with a mass of $125 \mathrm{GeV}$ is the lightest of the three neutral Higgs bosons predicted by a 2 HDM. The search focuses on the

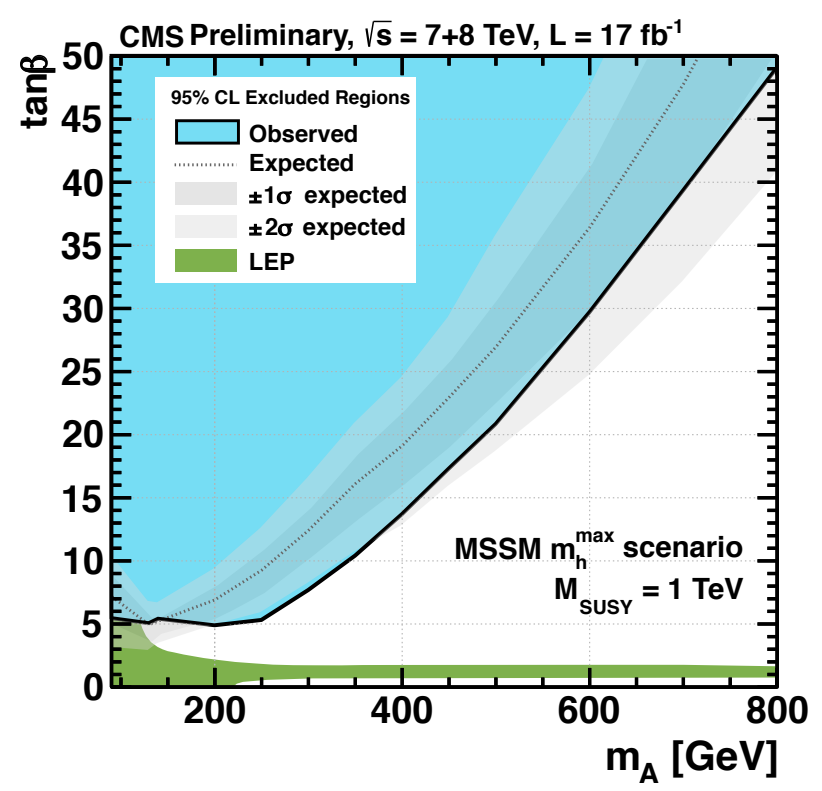

Fig. 5. CMS exclusion limits for $\Phi \rightarrow \tau \tau$ in the $m_{h}^{\max }$ scenario of the MSSM [13]. The LEP limit is shown as well.

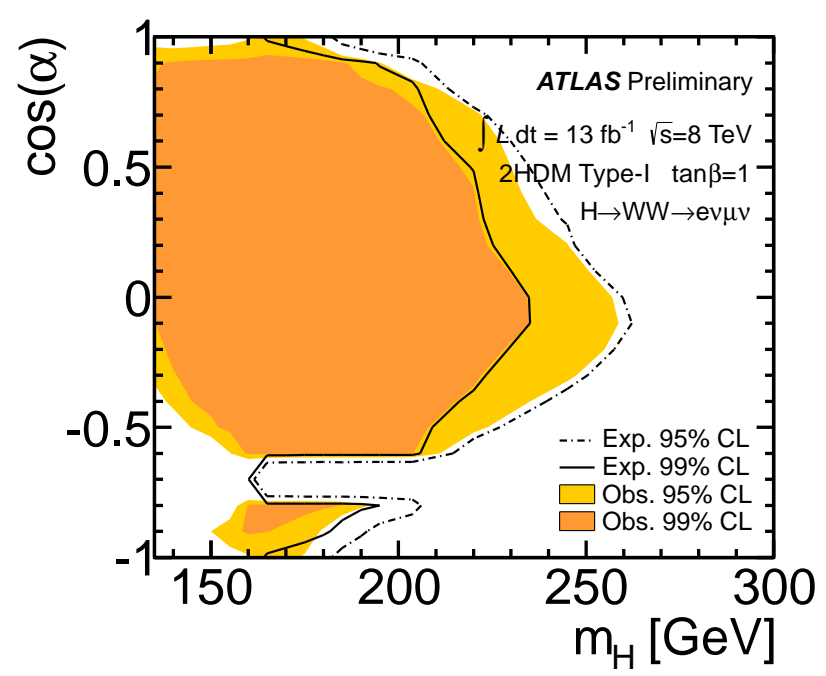

Fig. 6. ATLAS exclusion limits for $H \rightarrow W W$ in a type-I 2HDM for $\tan \beta=1[16]$.

$H \rightarrow W W \rightarrow e v \mu v$ decay mode with 0 or 2 additional jets and uses $13 \mathrm{fb}^{-1}$ of $8 \mathrm{TeV}$ data [16]. The preselection is in common with the SM $H \rightarrow W W$ search. To maximize sensitivity, an artificial neural net is employed using kinematic information about the event. No evidence for a Higgs boson in the mass range of $135-300 \mathrm{GeV}$ is found. Exclusion limits are set for type-I and type-II 2HDM for a set of fixed values of $\tan \beta$ ranging from 1 to 50 in the $m_{H}$-cos $\alpha$ plane (an example is shown in figure 6). Assuming that other Higgs bosons or additional particles are too heavy to interfere the tree-level cross section depends only on these three unknown parameters. 


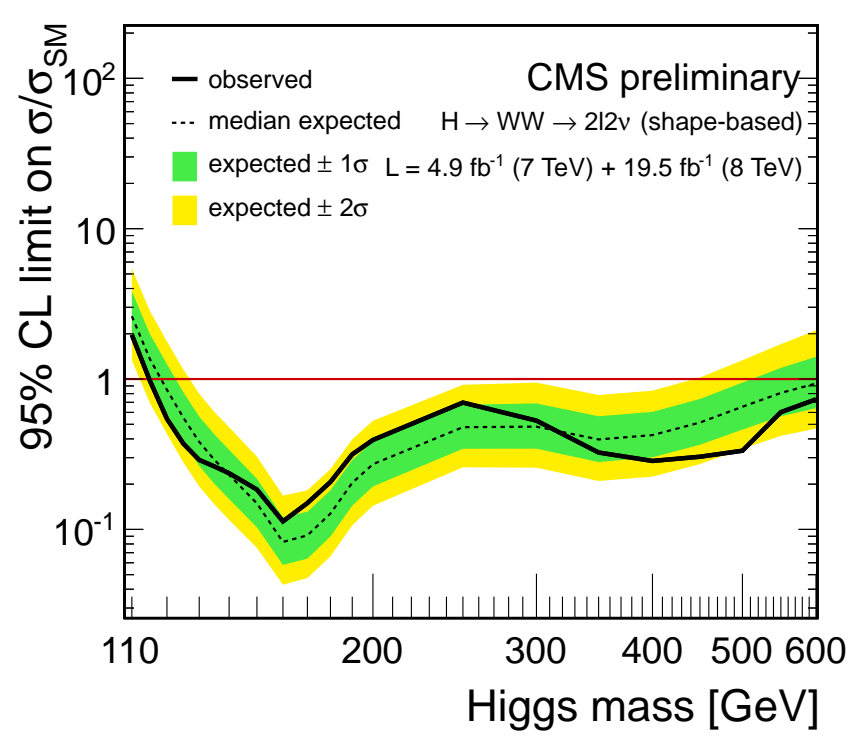

Fig. 7. CMS exclusion limit for a heavy Higgs boson decaying via $H \rightarrow W W \rightarrow l v l v$ [17]. Here, an SM Higgs boson with $m_{H}=125 \mathrm{GeV}$ has been added as background process. The limit is given in units of the expected SM Higgs cross section as a function of the heavy Higgs boson mass.

\section{Generic and exotic Higgs boson searches}

\subsection{Heavy Higgs, $H \rightarrow V V$}

Several BSM models are consistent with a Higgs boson with $m=125 \mathrm{GeV}$ and additional heavy Higgs bosons; in the most simple case, only one additional real Higgs singlet is added to the Standard Model leading to a second heavy Higgs boson which can also be SM-like. Such Higgs bosons are searched for in decays to weak bosons, $H \rightarrow W W$ and $H \rightarrow Z Z$, and the analysis strategy closely follows the SM Higgs boson searches.

CMS uses up to $25 \mathrm{fb}^{-1}$ of $7 \mathrm{TeV}$ and $8 \mathrm{TeV}$ data in searches for $H \rightarrow W W \rightarrow l v l v$ [17] and $H \rightarrow W W \rightarrow$ $l v q q$ [18], ATLAS $5 \mathrm{fb}^{-1}$ of $7 \mathrm{TeV}$ data in the $l v l v$ final state [19]. No significant excess is observed, and an additional heavy Higgs boson with SM couplings is excluded up to a mass of $m_{H}=600 \mathrm{GeV}$; however, a cross section times branching ratio less than $10-70 \%$ (depending on $m_{H}$ ) of the SM prediction is still allowed, see figure 7.

Recent heavy Higgs boson searches in the $H \rightarrow Z Z$ channel focus on the final states $2 l 2 q$ [20], $2 l 2 v$ [18] and $4 l+2 l 2 \tau$ [21] for CMS, and $4 l$ [22] for ATLAS. The data agree well with the SM expectation, and the most stringent limits can be set in the $4 l+2 l 2 \tau$ analysis and are shown in figure 8. A second Higgs boson with SM couplings is excluded for the mass range $m_{H}=(130-827) \mathrm{GeV}$ and

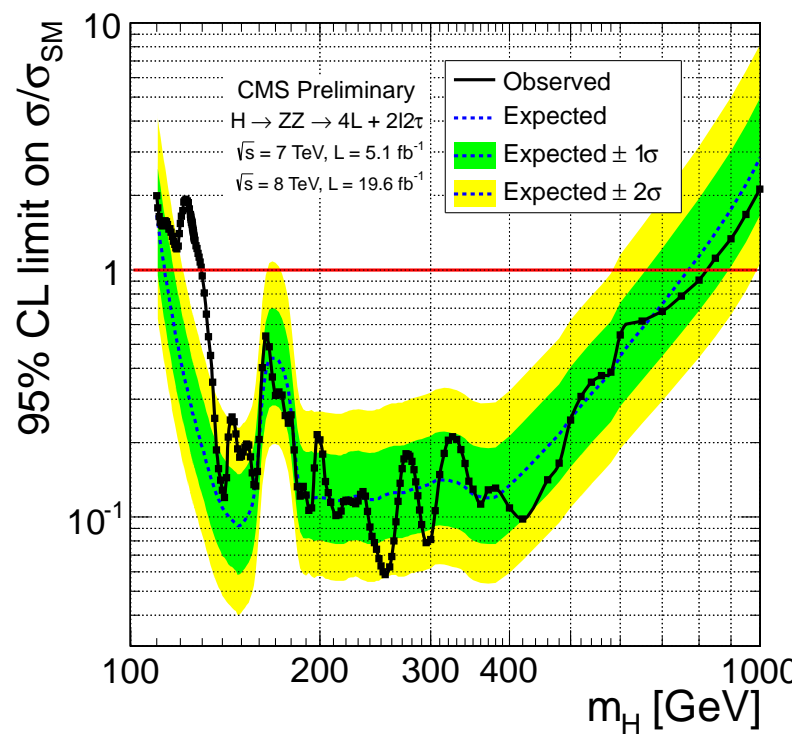

Fig. 8. CMS exclusion limit for a Higgs boson decaying via $H \rightarrow$ $Z Z \rightarrow 4 l+2 l 2 \tau$ [21]. The limit is given in units of the expected SM Higgs cross section as a function of the Higgs boson mass.

for $m_{H}=(180-500) \mathrm{GeV}$, a cross section times branching ratio larger than $20 \%$ of the SM prediction is excluded.

\subsection{Invisible Higgs, $Z H$}

The ATLAS search for invisible Higgs boson decays [23] considers Higgs bosons produced in association with a $Z$ boson in $18 \mathrm{fb}^{-1}$ of $7 \mathrm{TeV}$ and $8 \mathrm{TeV}$ data. To tag the event, an electron or muon pair consistent with the $Z$ boson mass is required. Events with additional leptons or jets are rejected. The missing transverse energy is used as final discriminant. No excess is observed, and assuming $m_{H}=125 \mathrm{GeV}$ and the SM cross section for $\mathrm{ZH}$ production, a branching ratio for invisible Higgs boson decays larger than $65 \%$ is excluded. In addition, upper limits between $30-7 \mathrm{fb}$ are set on the cross section times branching ratios for invisible Higgs boson decays in the mass range $m_{H}=(115-300) \mathrm{GeV}$, see figure 9.

\section{3 $H \rightarrow a_{0} a_{0} \rightarrow 4 \gamma$}

ATLAS uses $4.9 \mathrm{fb}^{-1}$ of $7 \mathrm{TeV}$ data to search for Higgs boson decays to very light CP-odd scalars $\left(m_{a_{0}}\right.$ of a few hundred $\mathrm{MeV}$ ) which in turn decay to photon pairs [24]. This is motivated by composite-Higgs models or the NMSSM but the results are interpreted in a model-independent way. Such a process leads to two highly collimated $a_{0} \rightarrow \gamma \gamma$ decays, which can mimick a diphoton event. The analysis requires such a diphoton pair using looser requirements for the shower shapes for photon identification than e.g. in the SM $H \rightarrow \gamma \gamma$ search due to two overlapping electromagnetic showers for the signal hypothesis. No significant excess is observed and upper limits of a few times $0.1 \mathrm{pb}$ on the cross section times branching ratios is set for the mass range $m_{H}=(110-150) \mathrm{GeV}$ and $m_{A}=100,200$, 


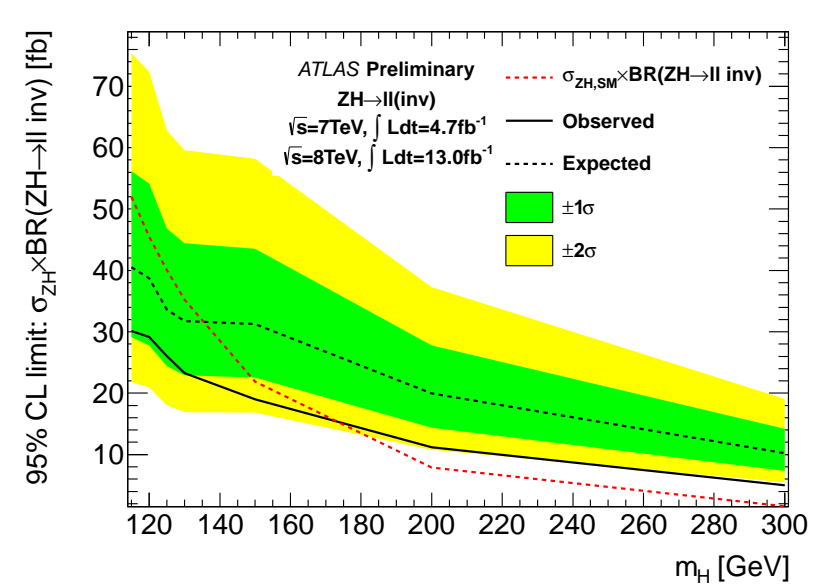

Fig. 9. ATLAS exclusion limit for invisible Higgs boson decays in $Z H$ production with $Z \rightarrow l l$ [23].

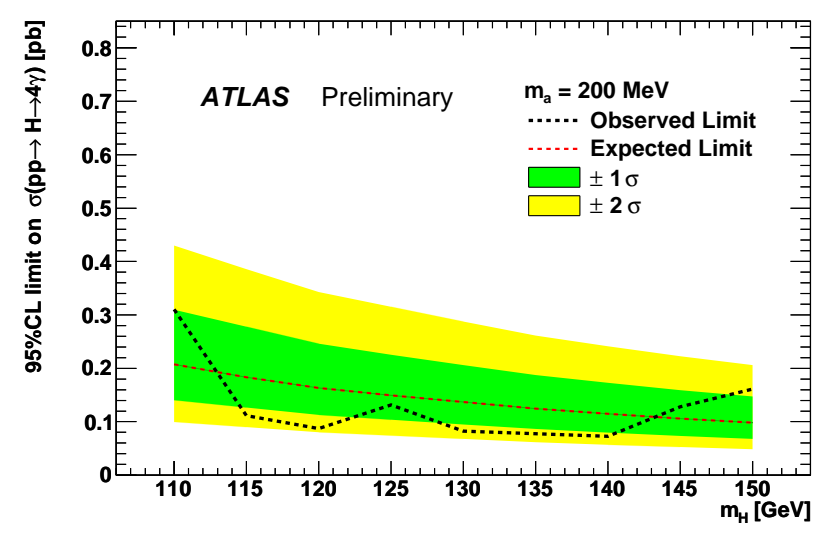

Fig. 10. ATLAS exclusion limit on the cross section times branching ratio for the process $H \rightarrow a_{0} a_{0} \rightarrow 4 \gamma$ with $m_{a_{0}}=200$ $\mathrm{MeV}$. Limits for other $m_{a_{0}}$ masses can be found in reference [24].

or $400 \mathrm{MeV}$. The limit for $m_{A}=200 \mathrm{MeV}$ is shown in figure 10 .

\subsection{Doubly-charged Higgs $\Phi^{ \pm \pm}$}

Both ATLAS and CMS use about $5 \mathrm{fb}^{-1}$ of $7 \mathrm{TeV}$ data to search for doubly-charged Higgs bosons in decays to same-sign lepton pairs $e^{ \pm} e^{ \pm}, e^{ \pm} \mu^{ \pm}, \mu^{ \pm} \mu^{ \pm}$. CMS, in addition, also looks for all possible combinations with tau leptons. The CMS analysis [25] investigates both pair production of and associated production with a singlycharged Higgs boson. The data agree with the SM expectation and upper limits on the cross section times branching ratio for $m_{\Phi^{ \pm \pm}}=130-500 \mathrm{GeV}$ are reported for different assumptions on the branching ratio of the doubly-charged Higgs boson. This result is translated in lower bounds on $m_{\Phi^{ \pm \pm}}$, see figure 11 for an example. For a branching ratio of 1 for the different lepton pair decays, doubly-charged

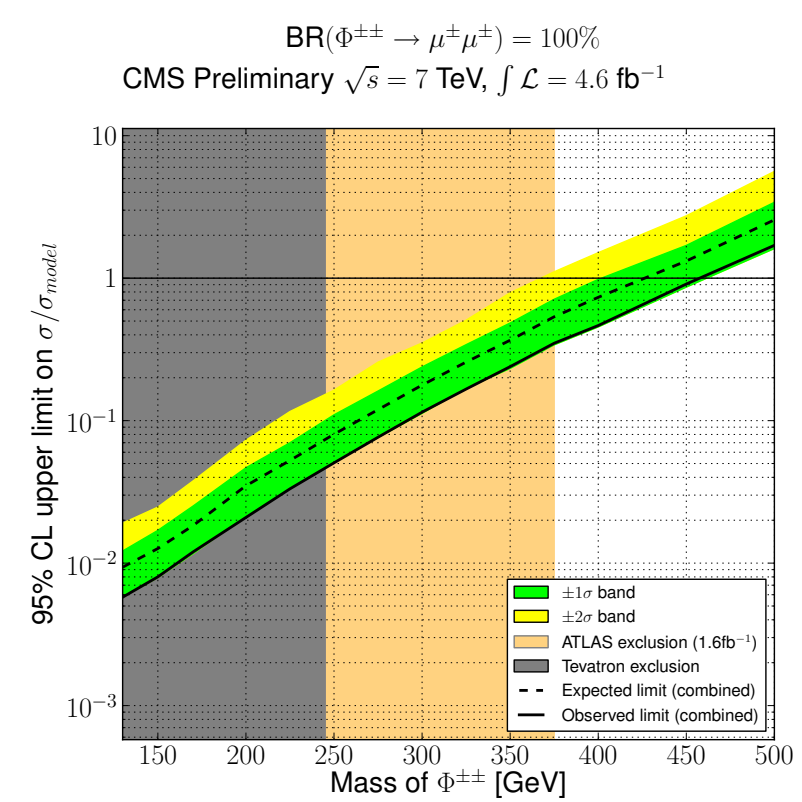

Fig. 11. CMS exclusion limit for doubly charged Higgs bosons [25]. The combined limit assuming $\operatorname{BR}\left(\Phi^{ \pm \pm} \rightarrow\right.$ $\left.\mu^{ \pm} \mu^{ \pm}\right)=100 \%$ is shown. The limit is given in units of the expected $\Phi^{ \pm \pm}$cross section in the investigated type-II seesaw mechanism model.

Higgs boson masses between 204 and $459 \mathrm{GeV}$ can be excluded.

The ATLAS analysis [26] searches for a narrow peak in the mass spectrum of same-sign lepton pairs. No excess is observed and the resulting limits on the cross section times branching ratio lead to lower bounds of about $400 \mathrm{GeV}$ on the $\Phi$ mass, assuming pair producion and a branching ratio of 1 to the investigated final states.

\subsection{Higgs in fermiophobic and SM4 models}

CMS searches for Higgs bosons in SM-like models with a fourth generation of fermions using up to $10 \mathrm{fb}^{-1}$ of $7 \mathrm{TeV}$ and $8 \mathrm{TeV}$ data [27]. In the investigated benchmark scenario, the effective Higgs coupling to gluons is enhanced by a factor of 4-9 (depending on $m_{H}$ ) while the decay to two photons is almost entirely suppressed. The search thus focuses on Higgs bosons produced in $g g$-fusion and decaying to $W W, Z Z$ and $\tau \tau$. No significant excess compatible with the signal hypothesis is observed and when combining the channels considered, consequently the SM4 benchmark scenario is excluded for Higgs boson masses between $110 \mathrm{GeV}$ and $600 \mathrm{GeV}$ (see figure 12).

In fermiophobic models, the Higgs coupling to fermions is suppressed. The CMS study [27], using $10 \mathrm{fb}^{-1}$ of $7 \mathrm{TeV}$ and $8 \mathrm{TeV}$ data, thus focuses on Higgs boson decays to photons in 9 different categories. The analysis excludes a fermiophobic Higgs boson with a mass in the range $110-147 \mathrm{GeV}$. 


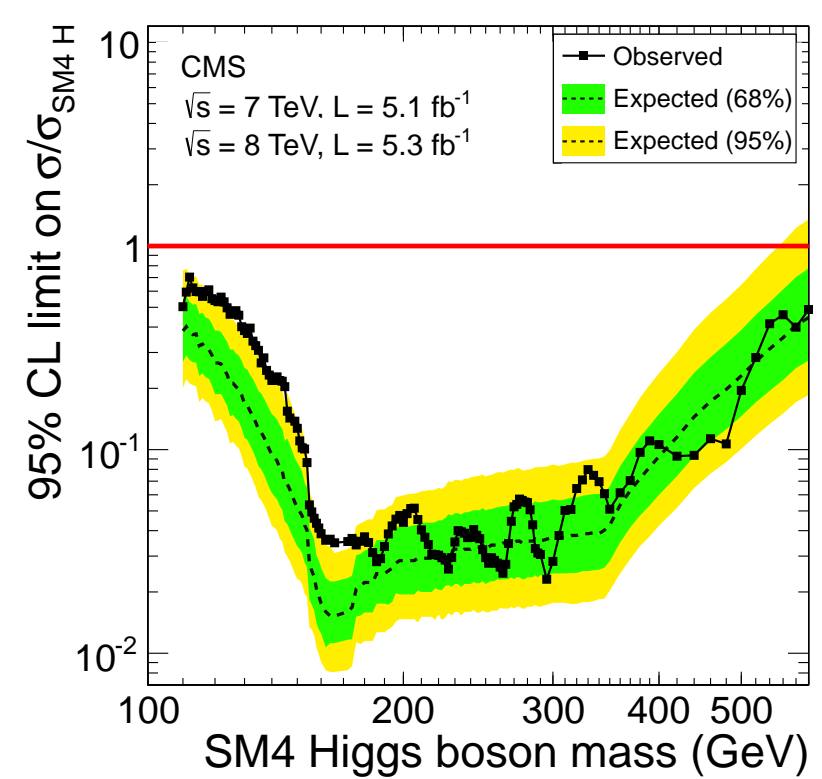

Fig. 12. CMS exclusion limit for the SM4 model using Higgs boson decays to $W W, Z Z$ and $\tau \tau$ [27]. The limit is given in units of the expected cross section in the investigated SM4 benchmark scenario.

\section{Conclusions}

A large number of BSM Higgs boson searches have been performed by the ATLAS and CMS experiments. No significant excess is observed, and various cross-section limits and exclusion regions for the parameter space of several models have been provided. In particular the MSSM is becoming heavily constrained: A new lower limit of $m_{A}>125 \mathrm{GeV}$ has been set for the $m_{h}^{\max }$ scenario (when including the region excluded by LEP), and for $125<m_{A}$ $[\mathrm{GeV}]<225$ only a $\tan \beta$ of about $2-5$ is still allowed. The limits extend up to $m_{A}=800 \mathrm{GeV}$ and $\tan \beta=50$. Strong cross section limits have been set for the existence of additional Higgs bosons with SM-like properties covering a large range of masses up to $1 \mathrm{TeV}$. Furthermore, general 2HDMs, the NMSSM as well as type-II seesaw mechanism models have been constrained.

Very few of these analyses have analysed the full Run-I LHC dataset, and in addition predictions of several well-motivated BSM models have not been experimentally tested at the LHC. It is thus still possible that BSM Higgs physics is hiding in the current data - and certainly, additional data collected at $\sqrt{s}=13 \mathrm{TeV}$ or higher at the LHC beginning from 2015 will greatly enhance the sensitivity to BSM Higgs bosons.

\section{References}

[1] ATLAS Collaboration, JINST 3, S08003 (2008).

[2] CMS Collaboration, JINST 3, S08004 (2008).

[3] L. Evans and P. Bryant (eds.), JINST 3, S08001 (2008).

[4] ATLAS Collaboration, Phys. Lett. B 716, 1 (2012).

[5] CMS Collaboration, Phys. Lett. B 716, 30 (2012).

[6] M. Carena et al, arXiv:1302.7033.

[7] ATLAS Collaboration, Eur. Phys. J. C 73, 2465 (2013).

[8] ATLAS Collaboration, JHEP 06, 039 (2012).

[9] ATLAS Collaboration, JHEP 03, 076 (2013).

[10] M. Carena et al, arXiv:hep-ph/9912223.

[11] CMS Collaboration, CMS-PAS-HIG-12-052, https: //cds.cern.ch/record/1502246.

[12] CMS Collaboration, Phys. Lett. B 722, 207 (2013).

[13] CMS Collaboration, CMS-PAS-HIG-12-050, https: //cds.cern.ch/record/1493521.

[14] CMS Collaboration, CMS-PAS-HIG-12-011, https: //cds.cern.ch/record/1453716.

[15] ATLAS Collaboration, JHEP 02, 095 (2013).

[16] ATLAS Collaboration, ATLAS-CONF-2013-027, http://cds.cern.ch/record/1525887.

[17] CMS Collaboration, CMS-PAS-HIG-13-003, http:// cds.cern.ch/record/1523673.

[18] CMS Collaboration, arXiv:1304.0213.

[19] ATLAS Collaboration, Phys. Lett. B 718, 391 (2012).

[20] CMS Collaboration, Phys. Lett. B 717, 70 (2012).

[21] CMS Collaboration, CMS-PAS-HIG-13-002, http:// cds.cern.ch/record/1523767.

[22] ATLAS Collaboration, ATLAS-CONF-2013-013, http://cdsweb.cern.ch/record/1523699.

[23] ATLAS Collaboration, ATLAS-CONF-2013-011, http://cds.cern.ch/record/1523696.

[24] ATLAS Collaboration, ATLAS-CONF-2012-079, http://cds.cern.ch/record/1460391.

[25] CMS Collaboration, Eur. Phys. J. C 72, 2189 (2012).

[26] ATLAS Collaboration, Eur. Phys. J. C 72, 2244 (2012).

[27] CMS Collaboration, arXiv:1302.1764. 